\title{
ACTITUDES Y CONOCIMIENTOS SOBRE LA PANDEMIA POR LA COVID-19 EN DOCENTES DE CANARIAS
}

\author{
Borja N. Santana-López@1,a, Yeray G. Santana-Padilla@1,b, Esteban G. Santana-Cabrera@2,c, \\ Gloria R. Ruiz-Rodríguez@2,d, Jesús M. González-Martín®11,e, Luciano Santana-Cabrera®1,f, \\ Grupo Multidisciplinar Educativo EDUCACOVID \\ ${ }^{1}$ Complejo Hospitalario Universitario Insular Materno Infantil de Gran Canaria, Las Palmas de Gran Canaria, Las Palmas, \\ España. \\ 2 Consejería de Educación, Las Palmas de Gran Canaria, Las Palmas, España. \\ ${ }^{a}$ Enfermero; ${ }^{b}$ enfermera, máster universitario en Ciencias de la Enfermería; ${ }^{c}$ docente en Educación Primaria, licenciado en \\ Geografía e Historia; ${ }^{\mathrm{d}}$ docente en Educación Primaria, máster universitario de Educación y TIC; ${ }^{\mathrm{e}}$ licenciado en Ciencias \\ Matemáticas; ${ }^{\mathrm{f}}$ médico especialista en Medicina Intensiva.
}

\section{RESUMEN}

Con el objetivo de estudiar las actitudes y conocimientos sobre la pandemia por la COVID-19 en el personal docente de la región de Canarias en España, se realizó un estudio transversal entre el 14 y el 18 de mayo de 2020, mediante la aplicación de un cuestionario virtual basado en un instrumento validado. Se incluyeron 1503 encuestas, en donde se describe que el personal docente no está dispuesto a trabajar si existe riesgo de infectarse en el centro educativo (76,6\%). No obstante, afirman que acudiría a trabajar si dispusieran de las medidas higiénicas $(69,2 \%)$ y de protección adecuadas $(67,0 \%)$. En conclusión, resulta pertinente garantizar las medidas preventivas necesarias para evitar el contagio en los centros educativos, propiciando la formación específica de los docentes en prevención primaria.

Palabras clave: Enfermedades Transmisibles; Infecciones por Coronavirus; Higiene; Docentes; Salud Laboral; Vigilancia; Educación en Salud; Promoción de la Salud (fuente: DeCS BIREME).

\section{TEACHERS' ATTITUDES AND KNOWLEDGE ABOUT THE COVID-19 PANDEMIC IN THE CANARY ISLANDS}

Citar como: Santana-López BN, SantanaPadilla YG, Santana-Cabrera EG, Ruiz-Rodríguez GR, González-Martín JM, Santana-Cabrera L, et al. Actitudes y conocimientos sobre la pandemia por la COVID-19 en docentes de Canarias. Rev Peru Med Exp Salud Publica. 2021;38(1):64-9. doi: https://doi. org/10.17843/rpmesp.2021.381.6312.

Correspondencia: Borja Nicolás Santana López; Calle San Borondón n.o 3, 35018, Las Palmas de Gran Canaria, España; borjaslp95@hotmail.es.

Recibido: $07 / 08 / 2020$ Aprobado: $23 / 09 / 2020$ En línea: 13/11/2020

\begin{abstract}
A cross-sectional study was carried out to determine the teachers' attitudes and knowledge about the COVID-19 pandemic in the Canary Islands region in Spain, between May 14 - 18, 2020. A virtual questionnaire based on a validated instrument was applied. We included 1,503 surveys, which showed that teachers are unwilling to work if there is risk of infection at the school (76.6\%). However, they state that they would go to work if they had the appropriate hygiene (69.2\%) and protection (67.0\%) measures. In conclusion, it is important to guarantee the necessary preventive measures to avoid contagion in educational centers, promoting specific training of teachers in primary prevention.
\end{abstract}

Keywords: Communicable Diseases; Coronavirus Infections; Hygiene; Faculty; Occupational Health; Surveillance; Health Education; Health Promotion (source: MeSH NLM).

\section{INTRODUCCIÓN}

Al 21 de septiembre del 2020, España era el noveno país del mundo con más casos de COVID-19(1), con un total de 671468 personas infectadas y 30663 fallecidos ${ }^{(2)}$. La primera medida tomada por la mayoría de los países para frenar la pandemia fue la suspensión de las actividades presenciales ${ }^{(3)}$. En España, más de 840000 docentes han tenido que usar las tecnologías de la información y comunicación (TIC) de forma improvisada y apresurada para no frenar el proceso educativo ${ }^{(4)}$.

La Organización Mundial de la Salud (OMS) establece recomendaciones para la reapertura de los centros educativos, entre ellas, la higiene de manos, limpieza y ventilación de aulas, y el uso de mascarillas. Así como la evaluación y gestión de estudiantes, profesores, otro personal escolar, medios de teleformación, etc. ${ }^{(5)}$ 
La planificación para la reapertura debería comenzar lo antes posible, estableciendo mecanismos para garantizar la continuidad del aprendizaje de forma segura, tanto de los trabajadores como del alumnado ${ }^{(6)}$. Sin embargo, no se puede obviar la posibilidad de contagios entre el alumnado y el profesorado durante el proceso de reapertura, con el consecuente cierre del centro educativo ${ }^{(7)}$. Por ello, el establecimiento de protocolos sociosanitarios, planes educativos de contingencia y un plan de digitalización en el centro son algunas de las propuestas que podrían minimizar el impacto de la COVID-19 en el ámbito educativo ${ }^{(7)}$.

Por lo expuesto anteriormente y debido al limitado número de estudios realizados en el personal docente, nos planteamos el objetivo de describir las actitudes y conocimientos sobre la pandemia por la COVID-19 en el personal docente de Canarias.

\section{EL ESTUDIO}

Se realizó un estudio transversal en la Comunidad Autónoma de Canarias en España, entre el 14 al 18 de mayo de 2020. La población diana fueron los docentes de cualquier institución educativa pública o privada, es decir, desde maestros de primaria hasta profesores universitarios. Se incluyeron aquellos docentes que firmaron el consentimiento informado y que respondieron todas las preguntas del cuestionario.

El instrumento de recolección está basado en un estudio publicado por el equipo de investigación en 2019 para conocer las actitudes y creencias del personal sanitario durante una hipotética pandemia por gripe ${ }^{(8)}$. Por tratarse de un cuestionario ad hoc, se realizó una adaptación al sector educativo; el equipo de investigación revisó que las preguntas no sean ambiguas o de difícil comprensión, y que se correspondan con el objetivo del estudio.

Para validar el instrumento, se utilizó la técnica Delphi en 19 expertos pertenecientes a distintos niveles del sector educativo, garantizando al menos dos participantes por cada nivel (centro infantil, centro de educación infantil y primaria, instituto de enseñanza secundaria, centro de educación especial, centro de formación profesional, centro universitario, otros). Estos expertos valoraron la idoneidad de cada uno de los ítems mediante una puntuación de 1 a 7 puntos en escala de Likert, donde 1 representó valores de desacuerdo y 7 valores de acuerdo. El consenso de los expertos se dio cuando el puntaje de cada ítem alcanzó una media mayor a 5 y una desviación típica menor a 2; de esta forma se excluyeron tres preguntas del cuestionario inicial. Posteriormente, para valorar el nivel de fiabilidad se calculó el alfa de Cronbach a través de las puntuaciones de los expertos, cuyo resultado fue un valor de 0,943 .

El cuestionario tuvo 16 preguntas, dividas en 4 secciones: actitudes (3), conocimientos (6), información sociodemográfica (5) e información laboral (2). El cuestionario virtual fue creado en

\section{MENSAJES CLAVE}

Motivación para realizar el estudio: La crisis mundial provocada por la COVID-19 obligó al cierre de las instituciones de enseñanza. El desconfinamiento y la vuelta a la actividad lectiva se convierte en un reto para la comunidad educativa, ya que el virus sigue circulando y existe la posibilidad de rebrotes. La intención de este estudio es corroborar si los docentes están preparados para este reto.

Principales hallazgos: Los docentes consideran que no deberían realizar formación presencial ante la ausencia de determinadas medidas de higiene y seguridad.

Implicancias: Es importante dotar de formación preventiva a la comunidad educativa para afrontar estas nuevas circunstancias de pandemia.

un formulario de Google y difundido a través de las redes sociales. Se solicitó el consentimiento informado de cada participante, lo que garantizó su anonimato en todo momento.

El análisis estadístico se realizó con el programa R Core Team 2020 versión 4.0.0. El análisis descriptivo de los datos se presentó con frecuencias y porcentajes. De una población aproximada de 25000 docentes se calculó un tamaño de muestra de 587 para que los datos fueran significativos al $95 \%$, con un error muestral del 4\%. Finalmente se obtuvieron 1509 encuestas, lo que nos permitió un análisis estadístico con un error muestral menor del $2 \%$, para un nivel de confianza del $99 \%$.

Este estudio cuenta con la aprobación de la Comité de Ética de la Investigación con Medicamentos del Hospital Universitario de Gran Canaria Dr. Negrín (CEI/CEIm HUGCDN).

\section{HALLAZGOS}

\section{Perfil del personal docente}

Se recibieron 1503 encuestas correctamente llenadas. La mayoría fueron mujeres (75,6\%), más de la mitad (52,5\%) tenían entre 31 y 50 años y convivían con su pareja e hijos (50,8\%). La mayoría eran docentes de institutos de enseñanza secundaria $(48,0 \%)$, y casi todos pertenecían al sector educativo público (91,2\%) (Tabla 1).

\section{Actitudes del personal docente}

La mayoría de los docentes no creen que pueda disponer, en el centro educativo, de las medidas de protección e higiene adecuadas contra la COVID-19 (73,3\%). Este hecho se relaciona con sus propias actitudes donde afirman que no están dispuestos a trabajar si hubiese un riesgo mayor del 
Tabla 1. Características personales y laborales de los docentes incluidos en el estudio $(n=1503)$.

\begin{tabular}{|c|c|c|}
\hline Características & $\mathbf{n}$ & $\%$ \\
\hline \multicolumn{3}{|l|}{ Características personales } \\
\hline \multicolumn{3}{|l|}{ Sexo } \\
\hline Hombre & 366 & 24,4 \\
\hline Mujer & 1137 & 75,6 \\
\hline \multicolumn{3}{|l|}{ Edad (años) } \\
\hline$<30$ & 66 & 4,4 \\
\hline $30-50$ & 789 & 52,5 \\
\hline$>51$ & 648 & 43,1 \\
\hline \multicolumn{3}{|l|}{ Provincia } \\
\hline Las Palmas & 1191 & 79,2 \\
\hline Santa Cruz de Tenerife & 312 & 20,8 \\
\hline \multicolumn{3}{|l|}{ Convivencia } \\
\hline Vive con su pareja e hijos & 764 & 50,8 \\
\hline Vive con su pareja sin hijos & 316 & 21,0 \\
\hline Vive solo/a & 204 & 13,6 \\
\hline Vive con padres u otros familiares & 219 & 14,6 \\
\hline \multicolumn{3}{|l|}{ Características laborales } \\
\hline \multicolumn{3}{|l|}{ Centro de actividad } \\
\hline Centro infantil 0-3 años & 36 & 2,4 \\
\hline $\begin{array}{l}\text { Centro de educación infantil y pri- } \\
\text { maria }\end{array}$ & 571 & 38,0 \\
\hline Instituto de enseñanza secundaria & 721 & 48,0 \\
\hline Centro de educación especial & 12 & 0,8 \\
\hline Centro de formación profesional & 55 & 3,6 \\
\hline Centro universitario & 24 & 1,6 \\
\hline Otro $^{\text {a }}$ & 84 & 5,6 \\
\hline \multicolumn{3}{|l|}{ Sector } \\
\hline Público & 1372 & 91,2 \\
\hline Privado-concertado ${ }^{b}$ & 77 & 5,2 \\
\hline Privado & 54 & 3,6 \\
\hline
\end{tabular}

a Escuela oficial de idiomas, centros de adultos, centros específicos de educación especial, colectivo de escuelas rurales

b Aquel centro privado que recibe a través de conciertos o acuerdos con el sistema educativo público para prestar sus servicios a la comunidad

usual en infectarse en el centro educativo (76,6\%) (Tabla 2). Además, en caso de que exista el riesgo añadido de infectar a sus familiares $(85,0 \%)$, probablemente tampoco acudirían al trabajo. Sin embargo, el 69,2\% iría a trabajar bajo medidas higiénicas y el $67,0 \%$ si contara con medidas de protección adecuadas.

Los docentes también manifestaron que no estarían dispuestos a trabajar si no existiera la posibilidad de mantener la distancia física de seguridad $(77,4 \%)$, si el alumnado no dispusiera del material de protección personal $(68,7 \%)$, si no hubiera solución hidroalcohólica dentro del aula $(65,0 \%)$, o si se le propusiera impartir docencia en grupos con ratios superiores $(80,0 \%)$ (Tabla 2 ).

En general, los docentes están en desacuerdo con impartir docencia presencial cuando existe un riesgo elevado de contagiarse ellos o su familia (94,9\%), este hecho es relevante ya que esta población convive en núcleos familiares de parejas e hijos. Se nota que los docentes anteponen la responsabilidad con su familia a sus deberes en el trabajo (92,5\%) (Tabla 3).

\section{Conocimientos del personal docente}

Se encontraron elevados porcentajes de conocimiento sobre las medidas adecuadas para protegerse contra la COVID-19 (Tabla 4). Sin embargo, el 52,0\% aún consideran que el uso de guantes es una medida adecuada de protección, el 91,6\% saben que no todas las mascarillas sirven para proteger contra la infección y que no solo han de cubrir la boca $(89,9 \%)$. Asimismo, el 68,4\% opinan que se debe mantener el uso de estas medidas de protección durante toda la jornada laboral.

Por otro lado, el 63,1\% sabe que para desinfectar las manos es necesario realizar la higiene con solución hidroalcohólica durante 20-30 segundos. No obstante, el 89,2\% opina que para evitar la contaminación de las manos es necesario lavarse con agua y jabón durante 40-60 segundos. Además, el 44,3\% opina que es necesario lavarse las manos con solución hidroalcohólica siempre que estén visiblemente sucias. Los docentes saben que la distancia física de seguridad se ha de mantener durante toda la jornada laboral (96,3\%).

Como medidas por mejorar, solo el $54,7 \%$ de los docentes indica que la mascarilla ha de cubrir la nariz, boca y barbilla y, solo el $26,8 \%$ indica que la medida más eficaz para evitar contaminar las manos es la solución hidroalcohólica.

\section{DISCUSIÓN}

En general, el personal docente no cree disponer en sus centros de trabajo de las medidas de protección e higiene y no están dispuestos a trabajar si existe riesgo de infectarse. No obstante, afirman que estarían dispuestos a trabajar si se les proporcionase las medidas de higiene y seguridad pertinentes. En lo relativo a los conocimientos se ha observado que conocen las medidas para protegerse del contagio, sin embargo, precisan de formación en materia de higiene y prevención de infecciones.

En relación con las actitudes, observamos una creencia general de desprotección, pues en su mayoría expresan no disponer de las medidas de protección e higiene en sus centros de trabajo. Esta impresión no es única de este gremio, 
Tabla 2. Actitudes del personal docente ante la pandemia por la COVID-19.

\begin{tabular}{|c|c|c|c|c|c|c|}
\hline \multirow{2}{*}{$\begin{array}{l}\text { Durante esta vuelta a la actividad docente tras el confinamiento por la COVID- } 19 \text { ¿con } \\
\text { qué probabilidad estaría más predispuesta/o a trabajar en las siguientes situaciones? }\end{array}$} & \multicolumn{2}{|c|}{ Probable } & \multicolumn{2}{|c|}{ No probable } & \multicolumn{2}{|c|}{ No sabe } \\
\hline & $\mathbf{n}$ & $\%$ & $\mathbf{n}$ & $\%$ & $\mathbf{n}$ & $\%$ \\
\hline Si hubiese un riesgo mayor de lo usual en infectarse en el centro educativo y caer enfermo & 195 & 13,0 & 1152 & 76,6 & 156 & 10,4 \\
\hline Si hubiera un riesgo mayor de lo usual en infectar a su familia & 161 & 10,7 & 1277 & 85,0 & 65 & 4,3 \\
\hline $\begin{array}{l}\text { Si tuviese que trabajar con las medidas higiénicas adecuadas (distancia mínima interpersonal, } \\
\text { ratio reducido de alumnos, solución hidroalcohólica) }\end{array}$ & 1040 & 69,2 & 208 & 13,8 & 255 & 17,0 \\
\hline $\begin{array}{l}\text { Si tuviese que trabajar con las medidas de protección adecuadas (mascarilla higiénica, mas- } \\
\text { carilla quirúrgica, batas, pantallas de protección...) }\end{array}$ & 1007 & 67,0 & 241 & 16,0 & 255 & 17,0 \\
\hline Si tuviese que trabajar sin las medidas de protección e higiene adecuadas & 161 & 10,7 & 1251 & 83,3 & 91 & 6,0 \\
\hline Si alguna de las personas con las que convive contrae la enfermedad & 119 & 7,9 & 1279 & 85,1 & 105 & 7,0 \\
\hline Si sus compañeros del centro educativo se hubieran infectado debido a la enfermedad & 204 & 13,6 & 1032 & 68,7 & 267 & 17,7 \\
\hline Si no existiera la posibilidad de mantener la distancia física de seguridad entre el alumnado & 188 & 12,5 & 1163 & 77,4 & 152 & 10,1 \\
\hline $\begin{array}{l}\text { Si no hubiera o existiera la posibilidad de mantener la distancia física de seguridad entre } \\
\text { profesorado y alumnado }\end{array}$ & 189 & 12,6 & 1163 & 77,4 & 150 & 10,0 \\
\hline Si no hubiera la posibilidad de disponer de solución hidroalcohólica dentro del aula & 280 & 18,6 & 977 & 65,0 & 246 & 16,4 \\
\hline Si no hubiera la posibilidad de disponer de solución hidroalcohólica en las zonas comunes & 247 & 16,5 & 1019 & 67,8 & 236 & 15,7 \\
\hline Si el alumnado pudiera disponer de material de uso personal & 952 & 63,4 & 216 & 14,3 & 335 & 22,3 \\
\hline Si el alumnado no pudiera disponer de material de uso personal & 182 & 12,1 & 1032 & 68,7 & 289 & 19,2 \\
\hline $\begin{array}{l}\text { Si le propusiera/pidiera impartir un mayor número de sesiones de docencia directa al alum- } \\
\text { nado }\end{array}$ & 373 & 24,8 & 711 & 47,3 & 419 & 27,9 \\
\hline Si se le propusiera/pidiera impartir docencia en grupos con ratios superiores & 170 & 11,3 & 1202 & 80,0 & 131 & 8,7 \\
\hline $\begin{array}{l}\text { Si se le pidiera impartir docencia a grupos en los que el alumnado requiera una mayor cer- } \\
\text { canía (alumnado con NEE/NEAE) }\end{array}$ & 430 & 28,6 & 709 & 47,2 & 364 & 24,2 \\
\hline
\end{tabular}

NEE/NEAE: Necesidades educativas especiales / necesidades específicas de apoyo educativo.

se describieron resultados similares en una encuesta nacional sobre las condiciones de trabajo de las enfermeras y enfermeros de España, durante la crisis sanitaria de la COVID-19 ${ }^{(9)}$.

Actualmente, los niños representan una baja proporción del total de los casos notificados, ya que permanecen mayoritariamente asintomáticos o desarrollan de forma leve la enfermedad ${ }^{(10)}$. La preocupación de los docentes sobre la trasmisión de la COVID-19 podría provocar absentismo ante determinadas circunstancias descritas en los ítems explorados, similares conclusiones se han descrito en un estudio similar ${ }^{(8)}$.

El personal docente debería utilizar mascarillas higiénicas que cumplan con los estándares específicos de calidad UNE 0064 y $0065^{(11)}$. No obstante, la OMS sentencia que un uso inadecuado de las mascarillas, lejos de reducir el riesgo de contagio, podría

Tabla 3. Actitudes del personal docente ante la pandemia por la COVID-19.

\begin{tabular}{|c|c|c|c|c|}
\hline \multirow{2}{*}{ Por favor, indique si tiende a estar de acuerdo o en desacuerdo con las siguientes afirmaciones } & \multicolumn{2}{|c|}{ De acuerdo } & \multicolumn{2}{|c|}{ En desacuerdo } \\
\hline & $\mathbf{n}$ & $\%$ & $\mathbf{n}$ & $\%$ \\
\hline $\begin{array}{l}\text { El personal docente tiene el deber de impartir docencia presencialmente, incluso cuando hay riesgos elevados } \\
\text { de infectarse ellos mismos o su familia. }\end{array}$ & 76 & 5,1 & 1427 & 94,9 \\
\hline $\begin{array}{l}\text { El personal docente al igual que el sanitario debe tener prioridad frente a la población general para ser diag- } \\
\text { nosticado y recibir tratamiento durante la desescalada. }\end{array}$ & 211 & 14,0 & 1292 & 86,0 \\
\hline $\begin{array}{l}\text { Se debe permitir que el personal docente se niegue a trabajar con, o cerca de, personas con síntomas respira- } \\
\text { torios. }\end{array}$ & 279 & 18,6 & 1224 & 81,4 \\
\hline $\begin{array}{l}\text { El personal docente que se niegue a desempeñar sus funciones durante esta época de crisis sanitaria debe ser } \\
\text { sancionado de alguna manera. }\end{array}$ & 220 & 14,6 & 1283 & 85,4 \\
\hline La responsabilidad en el trabajo está por encima de sus deberes familiares. & 112 & 7,5 & 1391 & 92,5 \\
\hline $\begin{array}{l}\text { El personal docente que tenga a su cargo alumnado en riesgo sanitario (inmunodeprimidos, enfermedad } \\
\text { crónica, etc.) podrá negarse a trabajar en caso de no tener las medidas adecuadas de protección e higiene. }\end{array}$ & 1415 & 94,2 & 88 & 5,8 \\
\hline
\end{tabular}


Tabla 4. Conocimientos del personal docente ante la pandemia por la COVID-19.

\begin{tabular}{|c|c|c|c|c|c|c|}
\hline \multirow{2}{*}{ Preguntas } & \multicolumn{2}{|c|}{ Sí } & \multicolumn{2}{|c|}{ No } & \multicolumn{2}{|c|}{ No sabe } \\
\hline & n & $\%$ & n & $\%$ & n & $\%$ \\
\hline \multicolumn{7}{|l|}{ Para protegerse contra el COVID-19 es necesario: } \\
\hline Lavado de manos con agua y jabón & 1490 & 99,2 & 8 & 0,5 & 5 & 0,3 \\
\hline Higiene de manos con solución hidroalcohólica & 1360 & 90,5 & 110 & 7,3 & 33 & 2,2 \\
\hline Mascarilla & 1395 & 92,8 & 68 & 4,5 & 40 & 2,7 \\
\hline Distanciamiento social & 1492 & 99,3 & 5 & 0,3 & 6 & 0,4 \\
\hline Aislamiento social & 527 & 35,1 & 820 & 54,5 & 156 & 10,4 \\
\hline Uso de guantes & 782 & 52,0 & 590 & 39,2 & 131 & 8,8 \\
\hline \multicolumn{7}{|l|}{ Responda a cada una de estas cuestiones marcando la respuesta que mejor refleje su opinión: } \\
\hline Todas las mascarillas sirven para proteger frente al COVID-19 & 63 & 4,2 & 1377 & 91,6 & 63 & 4,2 \\
\hline Es necesario llevar siempre guantes & 191 & 12,7 & 1237 & 82,9 & 75 & 4,4 \\
\hline Únicamente es necesario cubrir la boca con la mascarilla & 108 & 7,1 & 1352 & 89,9 & 43 & 3,0 \\
\hline $\begin{array}{l}\text { Es necesario lavarse las manos con solución hidroalcohólica siempre que las manos estén } \\
\text { visiblemente sucias }\end{array}$ & 667 & 44,3 & 767 & 51,0 & 69 & 4,7 \\
\hline Es necesario lavarse con agua y jabón durante 40-60 segundos & 1341 & 89,2 & 109 & 7,3 & 53 & 3,5 \\
\hline Es necesario mantener durante toda la jornada laboral mascarilla & 1029 & 68,4 & 336 & 22,5 & 138 & 9,1 \\
\hline Es necesario mantener durante toda la jornada laboral la distancia física de seguridad & 1448 & 96,3 & 41 & 2,7 & 14 & 1,0 \\
\hline Es necesario lavarse con solución hidroalcohólica durante 20-30 segundos & 949 & 63,1 & 381 & 25,4 & 173 & 11,5 \\
\hline $\begin{array}{l}\text { Es necesario desinfectar con lejía o sus derivados cada material del aula después de cada } \\
\text { clase }\end{array}$ & 1252 & 83,3 & 98 & 6,6 & 153 & 10,1 \\
\hline Es necesario mantener el aula ventilada en todo momento durante la jornada laboral & 1403 & 93,3 & 42 & 2,6 & 58 & 4,0 \\
\hline El alumnado puede compartir materiales y libros docentes durante las sesiones de clase & 30 & 2,0 & 1410 & 93,8 & 63 & 4,2 \\
\hline Se debe disponer de gel de solución hidroalcohólica en todas las aulas. & 1429 & 95,0 & 38 & 2,5 & 36 & 2,4 \\
\hline Es necesario que la comunidad educativa sepa hacer un correcto lavado de manos & 1491 & 99,2 & 6 & 0,4 & 6 & 0,4 \\
\hline \multicolumn{7}{|l|}{ ¿Qué superficie de la cara tiene que cubrir la mascarilla? } \\
\hline Boca & 2 & 0,1 & - & - & - & - \\
\hline Nariz y boca & 678 & 45,2 & - & - & - & - \\
\hline Nariz, boca y barbilla & 823 & 54,7 & - & - & - & - \\
\hline \multicolumn{7}{|l|}{ Usando la mascarilla ¿a quién protege? } \\
\hline Al propio individuo & 237 & 15,8 & - & - & - & - \\
\hline A las personas del entorno inmediato & 1266 & 84,2 & - & - & - & - \\
\hline \multicolumn{7}{|l|}{ ¿Qué medida es más eficaz para evitar contaminar las manos? } \\
\hline Higiene de manos con solución hidroalcohólica durante 20 segundos & 403 & 26,8 & - & - & - & - \\
\hline Lavado de manos con agua y jabón durante 20 segundos & 815 & 54,2 & - & - & - & - \\
\hline Usar guantes & 285 & 19,0 & - & - & - & - \\
\hline \multicolumn{7}{|l|}{ ¿Cuánta es la distancia física que ha de mantenerse entre personas para evitar el contagio? } \\
\hline 1 metros & 106 & 7,1 & - & - & - & - \\
\hline 2 metros & 1384 & 92,0 & - & - & - & - \\
\hline 3 metros & 13 & 0,9 & - & - & - & - \\
\hline
\end{tabular}

incluso aumentarlo ${ }^{(12)}$. Además, se deben aplicar todas las medidas destinadas para evitar la propagación de la pandemia ${ }^{(13)}$. Según la Ley 31/1995 de Prevención de Riesgos Laborales la administración pública de España debe garantizar a los trabajadores una protección eficaz en materia de seguridad y salud en el trabajo, además de formarles en el uso correcto de los equipos de protección ${ }^{(14)}$.

Los docentes probablemente no acudirían a su centro de trabajo si hubiera un riesgo mayor de infectarse y, por tanto, de contagiar a sus familiares. Este riesgo se ha demostrado en un estudio realizado por la Universidad de Granada donde se advierte que un grupo de alumnos compuesto por 20 niños tendría contacto con más de 800 personas después de solo dos días ${ }^{(15)}$.
Se han descrito algunas brechas de conocimiento entre los docentes, por lo cual será necesario instaurar medidas formativas. Destacamos que conocen las medidas básicas de higiene y protección a aplicar en los centros para evitar la infección y propagación del coronavirus ${ }^{(13)}$. Además, más de la mitad considera el uso de guantes como una medida de protección eficaz contra la COVID-19. Por lo tanto, habría que insistir en medidas de prevención de infecciones, como realizar una correcta higiene de manos y lograr normalizar esta práctica a toda la comunidad educativa ${ }^{(16)}$

El personal docente es clave para fomentar las medidas de promoción de la salud, por lo que se recomienda incluir profesionales sanitarios en el ambiente educativo para pro- 
porcionar a los docentes formación en materia preventiva, colaborando así en la adecuación de las medidas de higiene y protección en los centros, además de ayudar a gestionar los posibles contagios $^{(17)}$.

La mayor limitación del presente estudio es que la muestra abarca distintos tipos de población educativa, desde niños de guardería hasta universitarios, a pesar de ello las conclusiones generales nos animan a explorar las distintas características de cada uno de los distintos niveles educativos. Además, todos los encuestados pertenecen a una comunidad autónoma, lo que impide la extrapolación de los datos a otras regiones con características distintas fuera del ámbito educativo español. Por otro lado, al tratarse de un cuestionario anónimo y electrónico, se asume el sesgo de que alguno de los entrevistados no pertenezca al gremio de los docentes.

En conclusión, los docentes de Canarias están dispuestos a trabajar de forma presencial siempre que se les aseguren las medidas de higiene y seguridad en su centro de trabajo; sin

\section{REFERENCIAS BIBLIOGRÁFICAS}

1. World Health Organization (WHO). Coronavirus disease (COVID-19) Dashboard. [Internet]. Ginebra: WHO; 2020 [citado el 21 de septiembre de 2020]. Disponible en: https://covid19.who.int/table.

2. Centro de Coordinación de Alertas y Emergencias Sanitarias. Actualización $n^{\circ}$ 211. Enfermedad por el coronavirus (COVID-19). 21 de septiembre de 2020. [Internet]. Madrid: Ministerio de Sanidad; 2020 [citado el 21 de septiembre de 2020]. Disponible en: https://www.mscbs.gob.es/profesionales/saludPublica/ccayes/alertasActual/nCov/documentos/Actualizacion_211_COVID-19.pdf.

3. The World Bank. World Bank Education and COVID-19 [Internet] Washington: TWB; 2020 [citado el 11 de julio de 2020]. Disponible en: https://www.worldbank.org/en/data/interactive/2020/03/24/world-bankeducation-and-covid-19.

4. García-Peñalvo F, Corell A, Abella-García V, Grande M. La evaluación online en la educación superior en tiempos de la COVID-19. Education in the Knowledge Society. 2020; 21,12-26. doi: 10.14201/eks23013.

5. World Health Organization (WHO). Considerations for school-related publichealth measures in the context of COVID-19 [Internet]. Ginebra: WHO; 2020 [citado el 1 de agosto de 2020]. Disponible en: https://www.who.int/ publications/i/item/considerations-for-school-related-public-health-measures-in-the-context-of-covid-19.

6. UNICEF. La educación frente al COVID-19: Propuestas para impulsar el derecho a la educación durante la emergencia [Internet]. Nueva York: UNICEF; 2020 [citado el 25 de mayo de 2020]. Disponible en: https:// www.unicef.es/sites/unicef.es/files/educa/unicef-educa-covid19-propuestas-proteger-derecho-educacion-emergencia-0.pdf.

7. Fundación Cotec. COVID-19 y educación I: Problemas, respuestas y escenarios. [Internet]. Madrid: COTEC; 2020 [citado el 6 de mayo de 2020]. Disponible en: https://cotec.es/media/COTEC_COVID19_EDUCACION_problemas_respuestas_escenarios.pdf.

8. Santana-López BN, Santana-Padilla YG, Martín-Santana JD, Santana-Cabrera L, Escot-Rodríguez C. Creencias y actitudes de trabajadores sanitarios y estudiantes de enfermería de una región de España ante una pandemia de gripe. Rev Peru Med Exp Salud Pública. 2019; 36:481-6. doi: 10.17843/ rpmesp.2019.363.4371.

9. Sindicato de Enfermería SATSE. 5.500 enfermeras y enfermeros graves por la Covid 19. [Internet]. Madrid; 2020 [citado el 3 de septiembre de embargo, no creen tenerlas. En cuanto a los conocimientos, existen ciertas brechas educativas, las cuales podrían subsanarse con una adecuada formación en materia de prevención de infecciones.

Agradecimientos: Grupo Multidisciplinar Educativo EDUCACOVID: Manuel Montesdeoca Calderín (Universidad de Las Palmas de Gran Canaria); José Javier Rodríguez Vega (Instituto de Educación Secundaria Agustín Millares Sall); Gladys M. Suárez González (Instituto de Educación Secundaria Casas Nuevas); Carmen Terrón Álvarez (Centro de Educación Infantil y Primaria Esteban Navarro); Juan José Gil Méndez (Instituto de Educación Secundaria Las Huesas); Rafaela Martel Ojeda (Centro de Educación Infantil y Primaria Amelia Vega); Santiago Ortigosa López (Universidad Complutense de Madrid); Ana Carlier Pérez (Centro de Educación Infantil y Primaria Santa Bárbara); Jovita Pérez Rosales (Centro de Educación Infantil y Primaria Claudio De la Torre).

Financiamiento: Autofinanciado.

Conflictos de interés: Los autores declaran no tener conflictos de interés.

2020]. Disponible en: http://www.satse.es/comunicacion/sala-de-prensa/ notas-de-prensa/5.500-enfermeras-y-enfermeros-graves-por-la-covid-19.

10. Viner RM, Russell SJ, Croker H, Packer J, Ward J, Stansfield C, et al. School closure and management practices during coronavirus outbreaks including COVID-19: a rapid systematic review. Lancet Child Adolesc Health. 2020;4(5):397-404. doi: 10.1016/S2352-4642(20)30095-X.

11. Ministerio de Consumo. ¿Qué debes tener en cuenta al comprar una mascarilla? [Internet]. Madrid: MIC; 2020. [citado el 3 de septiembre de 2020]. Disponible en: https://www.mscbs.gob.es/profesionales/saludPublica/ ccayes/alertasActual/nCov-China/documentos/030520_GUIA_COMPRA_MASCARILLAS.pdf.

12. World Health Organization (WHO). Advice on the use of masks in the community setting in Influenza A (H1N1) outbreaks. [Internet]. Ginebra: WHO; 2020 [citado el 3 de septiembre de 2020] Disponible en: https:// www.who.int/influenza/preparedness/measures/Adviceusemaskscommunityrevised.pdf.

13. Santana-Cabrera L, Santana-Cabrera EG, Santana-López BN. Medidas a implantar en la vuelta a la escuela en período Covid-19. Rev Esp Salud Pública. 2020;94:e1-e2.

14. Ley 31/1995, de 8 de noviembre, de prevención de Riesgos Laborales [Internet]. Boletín Oficial del Estado. num. 269, de 10 de noviembre de 1995 [citado el 7 de marzo de 2020]. Disponible en: https://www.boe.es/buscar/ pdf/1995/BOE-A-1995-24292-consolidado.pdf.

15. La Vanguardia. Un aula con 20 niños tendrá contacto con más de 800 personas en dos días. İnternet]. Granada: La Vanguardia; 2020 [citado el 18 de julio de 2020]. Disponible en: https://www.lavanguardia.com/ vida/20200617/481822039593/aula-20-ninos-contacto-800-personas-inicio-escolar.html.

16. Stone TE, Kunaviktikul W, Omura M, Petrini M. Facemasks and the Covid 19 pandemic: What advice should health professionals be giving the general public about the wearing of facemasks?. Nurs Health Sci. 2020; 22 (2):33942. doi: 10.1111/nhs. 12724 .

17. Maughan ED. Building Strong Children: Why We Need Nurses in Schools. American Educator. 2020; 40(1):19-22. Disponible en: https://files.eric. ed.gov/fulltext/EJ1094215.pdf. 